\title{
The Use of Three-Dimensional Integrated Design System in Smart Substation Design
}

\author{
Xiangli Liu1, Xinju Guo1, Lin Chen², Yun Zhou², Chunwen Xin² \\ ${ }^{1}$ State Grid Henan Economic Research Institute, Zhengzhou, China \\ ${ }^{2}$ State Grid Nanyang Electric Power Supply Company, Nanyang, China \\ Email: 287752033@qq.com
}

Received January 2014

\begin{abstract}
The traditional power design, construction, has been able to meet the requirements, but it has not been established engineering data database support and the correlation between the data is poor, due to inadequate coordination among design professionals, design easily lead to mistakes, field problems, and design results failed in all aspects of the project to be fully utilized. With the technology of three-dimensional integrated design system penetrates in all walks of life, the introduction of three-dimensional integrated design system substation design solutions and the application of common devices of State Grid Corporation in recent years, which provide technical conditions for the application of virtual reality technology in substation design. According to its own characteristics of substation project, we now propose prospect on virtual reality technology in substation design application.
\end{abstract}

\section{Keywords}

Database; Substation Design; Virtual Reality Technology; 3D Integration

\section{Introduction}

With the rapid development of information technology, three-dimensional design has become an inevitable trend and universal demand in the development of power engineering design in the future [1]. As the core of digital intelligent substation, three-dimensional model must apply intelligent 3D design through full-featured three-dimensional integrated system that produces intelligent substation application, basing on the model case of main wiring and distribution systems of universal design in the State Grid Corporation, and depending on appropriate choice of generic engineering design, appropriate adjustments in the main wiring according to the project size, and then choose the direction of the line and stop the door outlet location to adjust the device type and relate engineering design parameters according to the State Grid Corporation common device interface allowing designers, automatic generated interval cross sectional view of the electrical distribution equipment and general layout of the floor plan. And it can be able to check the security of the device and calculate mechanical lightning protection of the wire and grounding design, equipment, materials and other statistical work. The substation space, equipment, accessories, appendages of type, size, spacing, and other information can be stored in the model program by the use of digital technology, the substation, intuitive and simple can be referenced and directly 
modified in a graphical interface [2]. Graphics and database are synchronically linked, plan, cross section, installation drawings, bill of materials, mentioning owned linkage map of all modifications all ensure consistency of engineering design.

Currently, the substation design uses AUTOCAD two-dimensional graphics platform basically, it is in computer-aided drafting stage, lacks professional software, the graphics workload is heavy, and duplication of effort are many. Modify the contents cannot be related to linkage, or it will prone to error. And it cannot achieve the relevant professional computing and the checksum, equipment and materials required artificial statistics, civil inventory data and drawings drawn by hand finishing.

\section{Theoretical and Practical Basis of the Project}

\subsection{The Brief Principle Description of the Research Contents of Projects}

The object of digital substation is mainly achieved by three-dimensional design management system, application of 3D design system and database management systems are based on the database, reflecting reality and logic relation of substation in 3D, 2D or 1D form, All figures, tables are mapped database data, chart and data are consistent. In order to achieve the construction and operation of the substation efficient management of the process, all information of each stage of the substation will be unified planning from design, procurement, manufacturing, construction, commissioning, operation, maintenance to decommissioning and being kept in a data warehouse untidily. To achieve establishment of such a large database at the design stage, we must rely on a comprehensive and efficient system of three-dimensional design and highly integrated database management system.

Three-dimensional integrated design system has the ability to build substation lifecycle information platform and can provide a digital substation for the owners, the basic works are as follows.

1) The unified identification system is established at the beginning of the project, began at the design through data warehouse platform, the information from engineering companies and engineering firms effectively delivered to the appropriate procurement, construction sector, and ultimately to operation and maintenance.

2) Substation design based on the 3D design system as the core design means, through a network of digital substation technology collaborative design, ensuring at the right time, right place, right information.

3) Using integrated Collaborative projects design, effective control versions and the corresponding workflow, centralized control and management of all technical documentation and publishing drawings and upgrades, making the design knowledge and achievements intact in a unified central database.

4) Schedule on-site and on-site construction and installation, commissioning, and centralized management of quality control information by integrating all of the owners and contractors substation project management and operational management system, forming the final figures substation, digital and knowledge management.

5) With the progress of the project, we will continue to accumulate data and maintain data consistency, establish a standardized model, data, drawings, documents. After the project is completed, the owners can finally submit to contain substation operation and maintenance data required for the future operation of the substation, digital substation maintenance management.

\subsection{The Practical Basis of Three-Dimensional Digital Substation}

Three-dimensional digital substation specific applications can be divided into three stages. In the design phase, in order to achieve a three-dimensional model with a powerful combination of information documents, form a complete three-dimensional visualization of plant information system. It needs so much information, such as an integrated three-dimensional model of a large number of design-related one-dimensional and two-dimensional design information, materials, finished products, and construction and operation and maintenance of the necessary information. In the infrastructure period, owners and construction unit use three-dimensional model to query design information and direct extraction inventory of a variety of design drawings, equipment and materials linking with model, provide detailed procurement information to the procurement system and simulate construction progress of the infrastructure and overhaul lifting reserved space, intuitive three-dimensional model to guide the construction, provide visual design communication services to remote construction site through INTERNET network

In the production run, the owners provide a standard project template through enterprise data warehouse, the 
engineering information of the substation is unified set in Interface of design and management, intelligence relation between all drawings, design content glance, drawing number is automatically generated according to volume, automated Summary table of generation engineering materials, volumes directory. Queries various stages of information as the core of the system, 3D model and database, auxiliary run operation, maintenance and operation of the auxiliary data maintenance, file management, training of personnel, and so on. The Project Workflow as Figure 1.

Three-dimensional model is able to achieve statistical calculation to generate digital model construction drawings [3]. Software can not only manifest real scenario of substation through three dimensions and a multiangle, making all the equipment, wires and special relationship of the structures as plain as print, but can also achieve the real lightning protection checking, precise materials statistics under a three-dimensional model. In addition, to better understand the design intent and design advantages, you can use three-dimensional animation in the substation to report the design, three-dimensional visual fully reflect the project design. Substation platform and three-dimensional design of digital transfer applications to query and retrieve information to provide

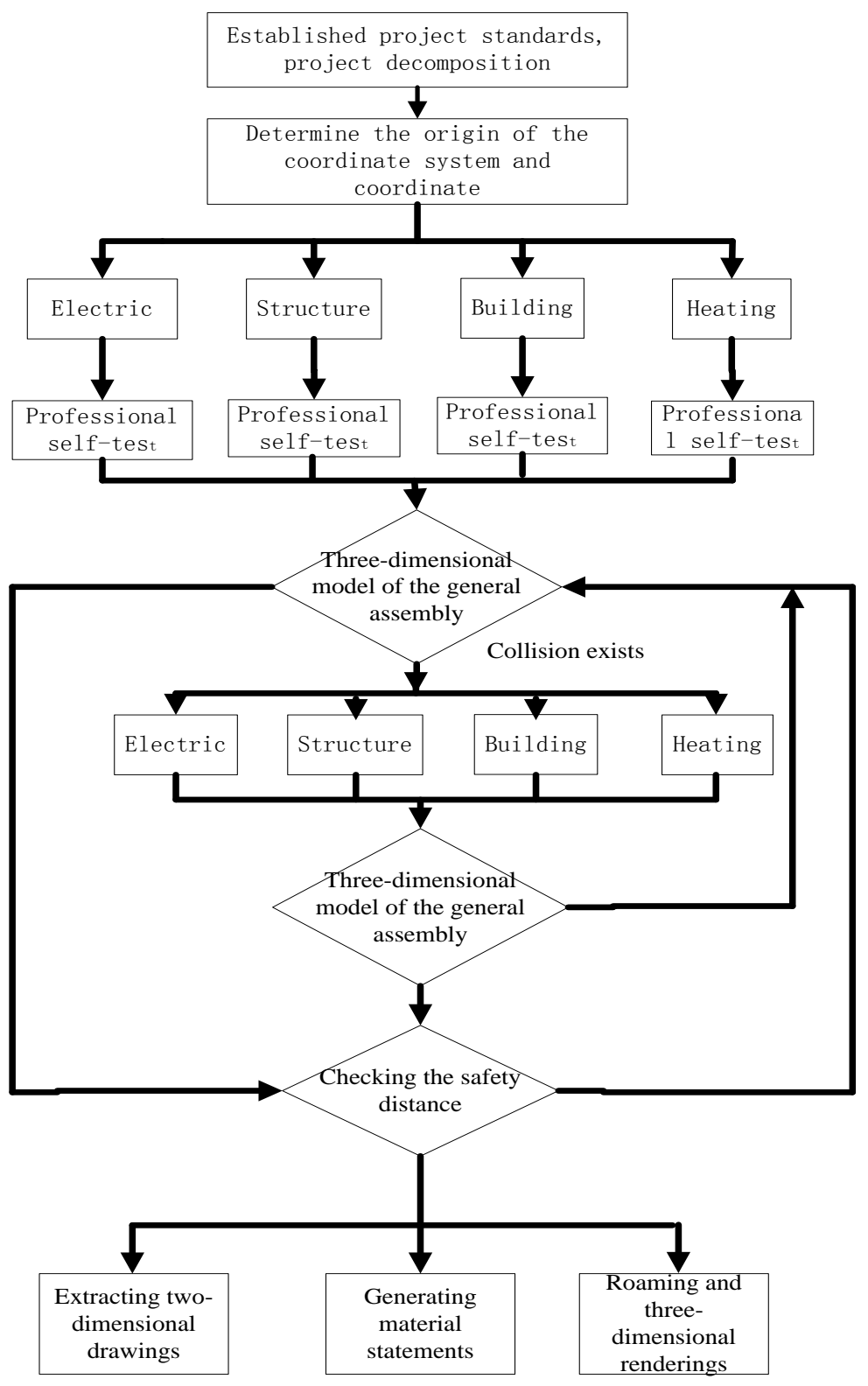

Figure 1. Project workflow. 
great convenience of owners and project management units checked without massive engineering drawings, simply via a web browser. Three-d Substation will be able to see realistic virtual dimensional model, all the engineering drawings and documents, all of the engineering data; just click on the model. It can be dispersed in water with automatic access to file data. Even without having to run around in a long institute, construction sites, run on-site inspection, just log network can view real-time design schedule, construction schedule, and procurement status, debug parameters, the actual operation of the device status, and annotate comments directly on the model. The Project Process as Figure 2.

\section{The Research Content and Implementation Plan}

Graphics technology accounts for a large share of the three-dimensional reality. How to bring real visual experience to the digital space? The key is how to simulate all the surfaces in the real world, this is the material, which includes lighting calculations, graphics such as texture blending by setting the texture layer's input and operation, the output can be used as an input for the next layer, can also be used as the end result, at her shows.

For two API of 3D hardware, OpenGL and Direct3D have their fixed rendering pipeline, which is the pipeline. Data of vertices such as position, normal, texture coordinates and so on will be provided to the pipeline, and then define the light source $[4,5]$, you can get an rendered image. In this particular rendering process, program designer has no ways to control. Starting from DirectX 8 and OpenGL extensions, programmable feature was introduced in the rendering pipeline, Graphics hardware began to support programmable features, so that the developers could write a small program and run it in the graphics card to control the rendering process. Functions of graphics hardware were developed from pipelined functional unit into two programmable units: vertex units and pixel units. Through programming about vertex units and pixel units, you can not only optimize the algorithm, but also implement functionality which could not be completed originally, such as distribute load between the vertex pipelines and pixel pipelines on their bearing capacity; through a custom algorithm, improve

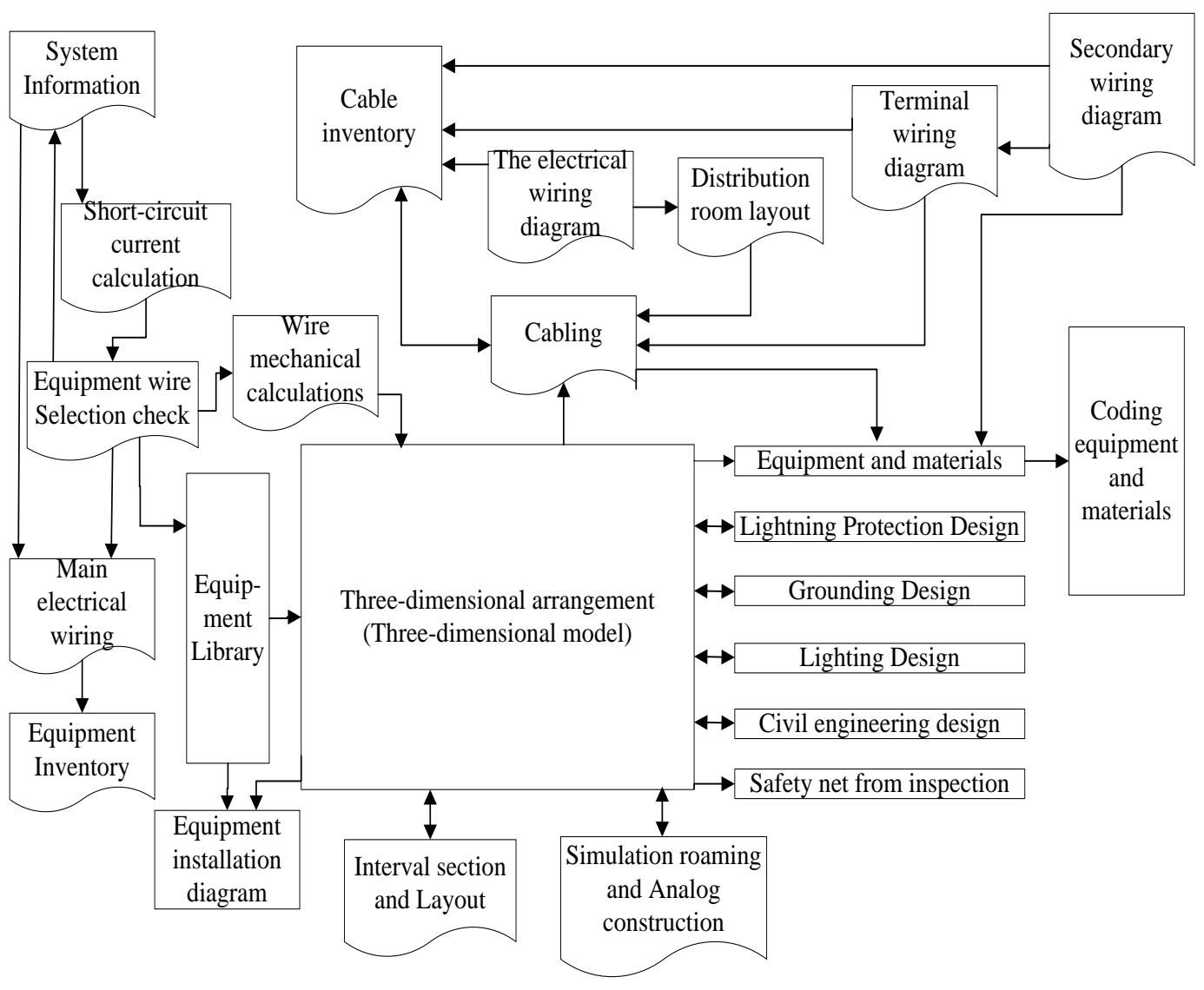

Figure 2. Project process. 
the algorithm of fixed-function, or even wholly create original algorithms. In the Microsoft DirectX, this piece of program code is called Shadier. In fact, the Shadier (render or shaded) are programs own the ability to manipulate 3D objects, and could be performed by the GPU. Through these programs, the programmers can get 3D graphical effects they wanted most. A 3D scene generally contains multiple Shadier. Some of this Shadier are responsible for processing the 3D object's surface, and some are responsible for handling screen objects which are cached provisionally. There are two kinds of Shadier, one is the peak level, known as the Vertex Shadier, replacing lighting part fixed rendering pipeline, programmers can control vertex transformation, lighting, and so on. The hardware unit which handles Vertex Shadberries called Vertex Shadier Processors (vertex processing units). The other one is the pixel-level called the Pixel Shader, it could replace grating section in the fixed rendering pipeline. Programmers can control the pixel color and texture sampling. Handling Pixel Shadier in hardware unit is called Pixel Shadier Processor (s-pixel processing unit). In the DirectX 9 Shadier, specialized editing and programming language is introduced-high level shadier language (HLSL: High-Level Shading Language). At the same time, file format is introduced to save code independently: file format. Fixes independent from programs, so users can change different Fx files and easily improve the Visual effect of the program without recompiling your program. Introducing programmable features are into the process of rendering is a revolution to real-time rendering; this gives developers more creative freedom, so users can create better effects. In this project, we use Shadier to define material of our model, on the one hand, this improved illumination algorithm and made texture-blending optimized, on the other hand, this in favor of modifying the material results easily in the future.

The program uses the modular structure. Modules are independent from each other. Standardized parameters are used to pass data. On the one hand this helps maintain a clear structure, it also facilitates the modification and maintenance. Module design process design can be split into data module design, menu module design, and interactive animation module design. Data module design mainly considers the texture processing, model making, the configuration in accordance with the target hardware, sets the format and resolution of textures, control the total numbers of polygon, budget allocation about polygon, the total model data format and structure. Design menu module's purpose is to reduce the difficulty of use and focus on the user experience. In the interactive animation module design, efficiency of algorithm is not required, but you should try to maintain a clear logical structure to ease the maintenance.

1) Typical engineering library management

Software uses a large network database to standardize the design of the typical universal design engineering, data commonly used by equipment manufacturers, spare libraries, equipment and other typical centralized management, to facilitate search and download, and ensure the security of the database through permission settings. Meanwhile platform database is open to members of the network. Members can simultaneously expand the open device database. A lot of graphics device parameters about equipment are stored in the equipment library. Through network management and permissions mechanism, digital design data and process management will be achieved.

2) 3-D Parametric modeling for equipment

By the platform's unique parametric three dimensional equipment models, just enter the key parameters, you will generate a planar, cross-section, the side view and solid of the device, and get "real time modifications to the three dimensional device" (parameter changes, equipment updated in real time). Software provides the fast and easy way to create three-dimensional device so that it could draw a three-dimensional shape easily.

3) The generation of general layout and section

Designer selects line outlet direction and pitted door location, while selects suitable of national grid General design programmer, platform will automatically generated preliminary electrical total plane, designer makes adjustment, platform will automatically generated eventually electrical total plane. This total plane contains large three dimensional database information, such as equipment parameter and size, architecture size, wire type, and the numbers of various of equipment. It can automatically generate a material table. For general layout, designers can cut it if he wants the distribution of the sectional view, and this can be generated by the power distribution unit cross section materials.

4) 3-D graph about substation lighting protection

Anti-thunder calculation is one process of plane figure draws for substation; platform provides three dimensional anti-thunders of draws. Designer only needs to place lightning rod in different places. So as long as designer types into the location and height about lightning rod, platform will automatically generate three dimen- 
sional anti-thunder figure, if there is architecture or equipment exposing outside the range, platform will find lightning rod not meet requires, then it will generate a perspicuous calculation book.

5) Powerful calculation function

Design platforms can do calculation features are: 1) short circuit current calculation and equipment selection, 2) dynamic grounding of substation meter grate, 3) wire tension calculation; 4) electricity calculation, and 5) secondary circuit relay protection setting calculation, and 6) cable and statistics. 7) Suspended tubes calculation software.

3-D model show as Figures 3-5.

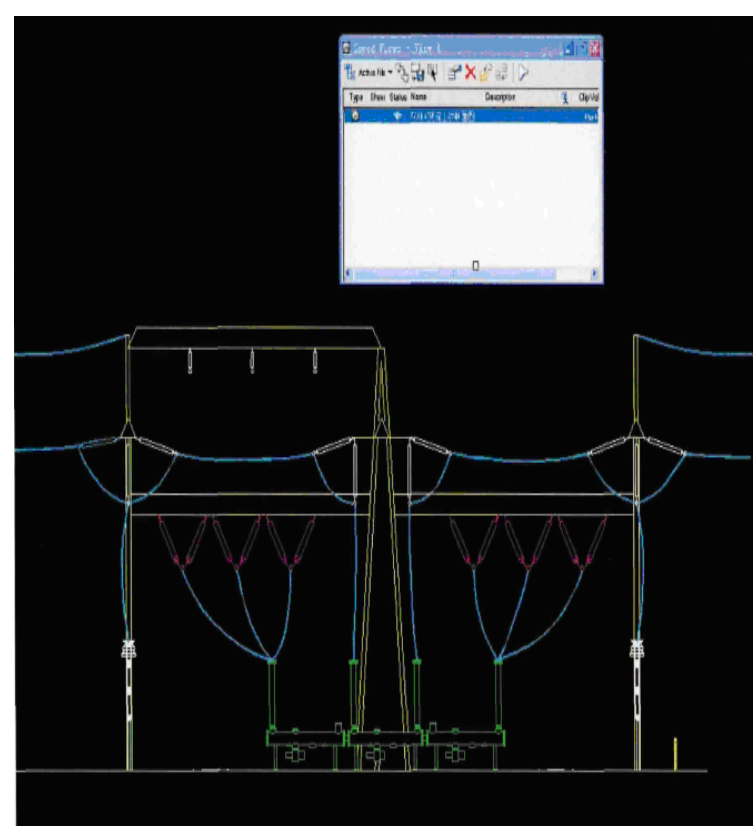

Figure 3. 3-Dgraphic model.

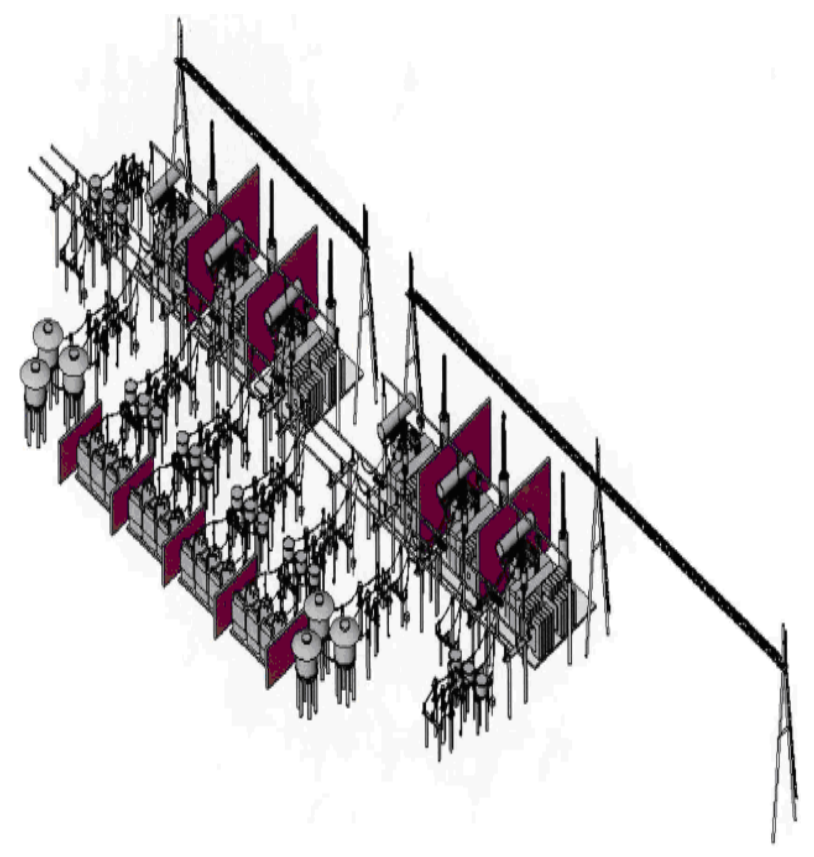

Figure 4. The main transformer and $35 \mathrm{kV}$ power distribution device model. 


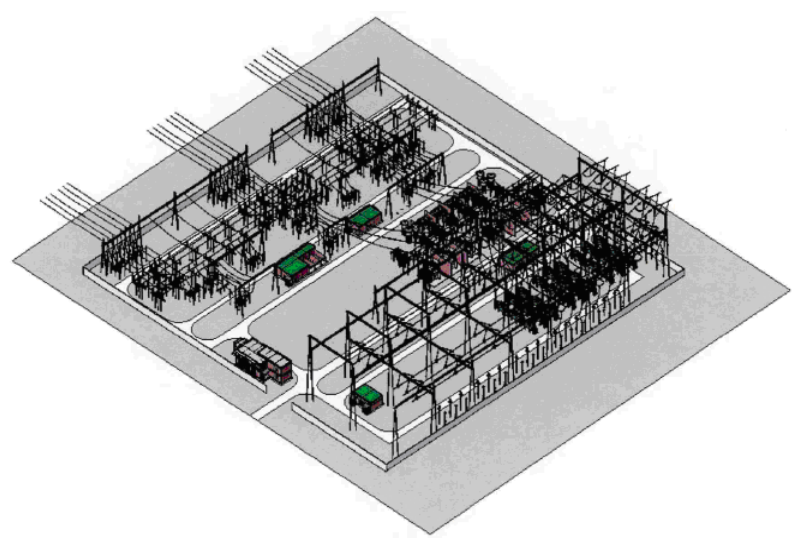

Figure 5. The whole 3-D model about total substation.

\section{Summary}

The method combines various data by using three-dimensional integration technology, builds virtual walk through system for substation design, provides design, planning, decision-making, with a visual reference, and provides a useful tool for further improvement programmer. In this method, three-dimensional reality technology for data integration, complex, professional data are visually reflected through virtual reality technology and this is more conducive to the exchange, identify problems, provide improvements, improve processing efficiency, and optimize the construction. This method is the most effective power substation design engineering aide.

Publishing design via the Internet, so that more people can learn about it and even participate in decisionmaking, benefit from universal knowledge of substation, and strengthen people's understanding of the substation. This can also increase the theoretical level of application environments, so that people can receive it more quickly.

\section{References}

[1] Pearson, A. (2005) The Real Toy the Virtual: Bergson and Delouse Jl. MLN, 120, 12-27.

[2] Marc, S. and Denis, L. (2010) Multiresolution Surface Modeling Based on Hierarchical Triangulation. Computer Vision and Image Understanding.

[3] Zhu, J. and Lubkeman, D.L. (2009) Object-Oriented Development of Software Systems for Power System Simulation. IEEE Transactions on Power Systems.

[4] Yukihiro, M. and Toshinori, Y. (2012) VR-Based Interactive Learning Environment for Power Plant Operator. Proceedings of the International Conference on Computers in Education.

[5] Huang, J. (2010) Construction of Dynamic Database System of Power System Elements. Journal of Electric Power, 12, 1-7. 